\author{
الممكنات الاقتصادية المتوقعة لتحقيق الامن الغذائي من القمح الليبي \\ زهرة صالح أحميدة ، نشوي عبد الحميد التطاوي ، منيرة طه الحاذق و الحسين عبد اللطيف الصيفي

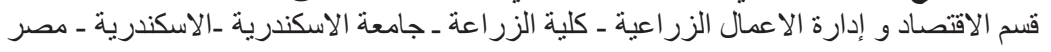

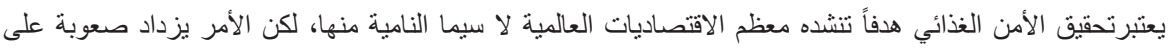

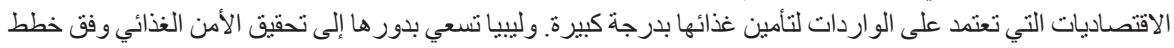

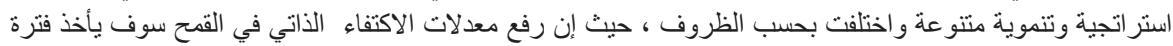

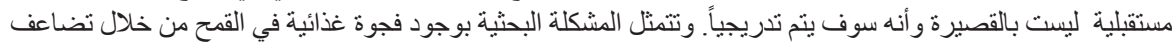

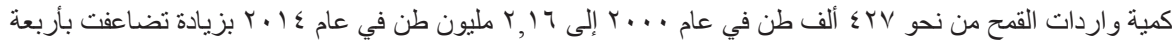

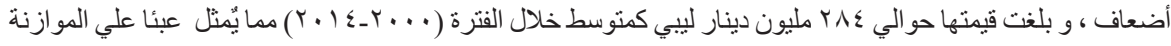

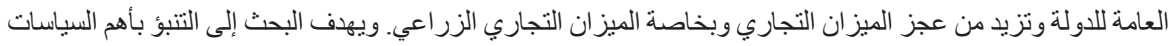

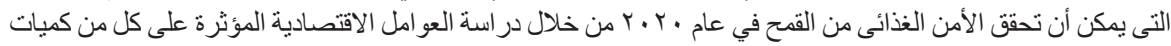

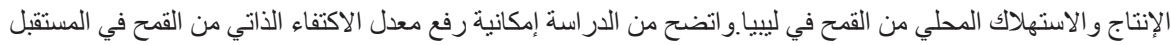

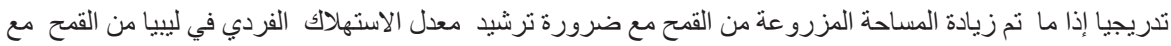

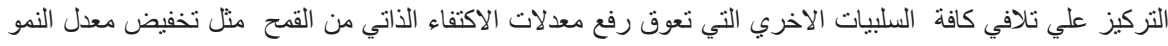

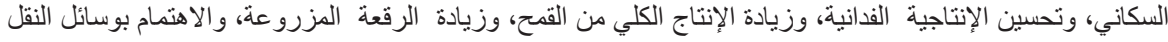

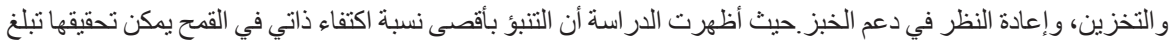

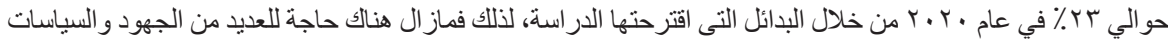

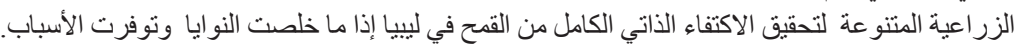

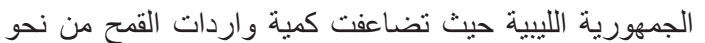

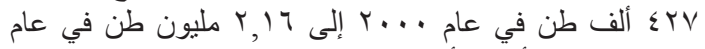

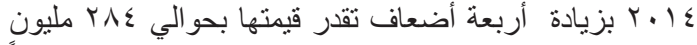

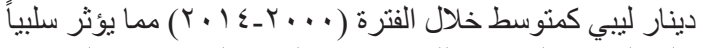

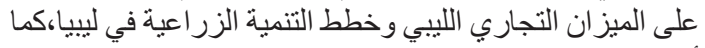

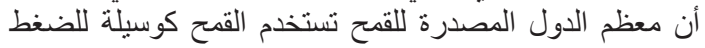

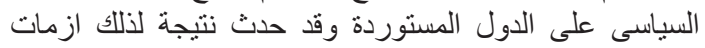
غذائية حادة فى ليبيا في عهود سابقة.

هدف البحث

استهدف البحث بصفة أساسية دراسة الممكنات الاقتصادية

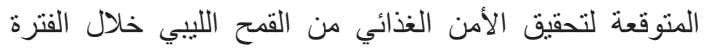

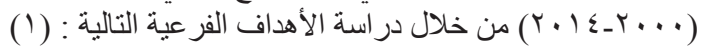

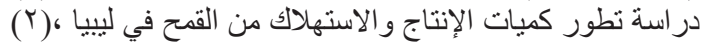

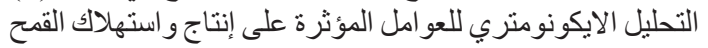

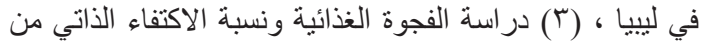

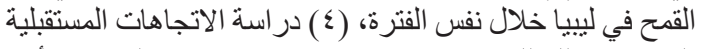

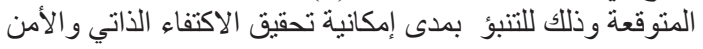

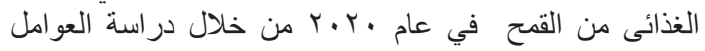

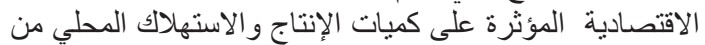
القمح في ليبيا.

\section{الطريقة البشثية ومصادر البيانات}

تم استخدام أسلوب التحليل الوصفي و الكمي من خلال الانحدار

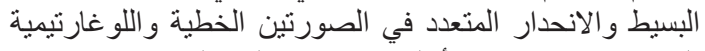

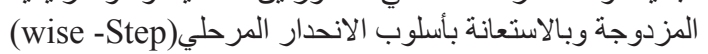

المقدمة

شاع استخدام مصطلح الأمن الغذائي منذ بداية السبعينيات للألالة

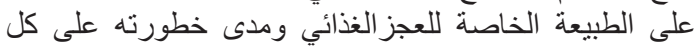

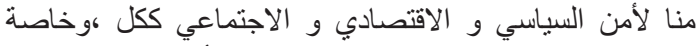
بالنسبة للدول النامية ،هذا وتتعدد تعاريف الأمن الأني الغذائي ولكنها

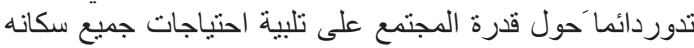

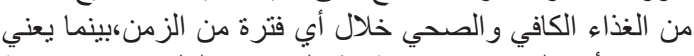

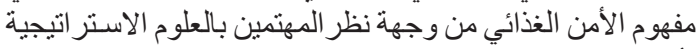

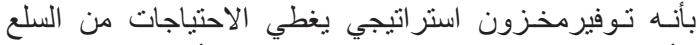

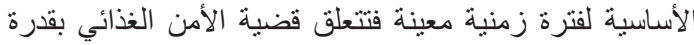

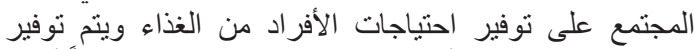

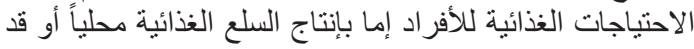

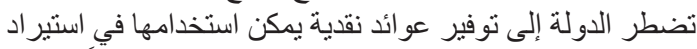

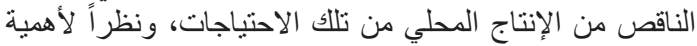
القمح كسلعة استر اتيجية في ليبيا حيث يعتبر من الإنيا أهم محاصيل

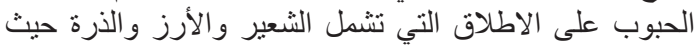

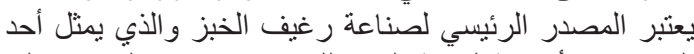

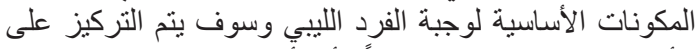

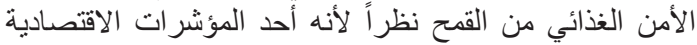
الهامة التي تبين الوضع الحالي للأمن الغذائي الليبي.

مشكلة البحث

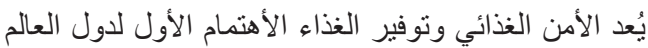

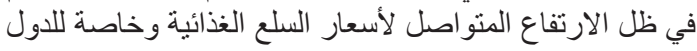

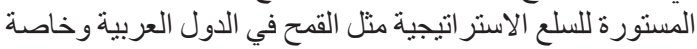

DOI :10.21608/jsas.2018.2425.1047

C2017 National Information and Documentation Center (NIDOC) 
r - الإنتاجية الإكتارية من القمح: تذبذبت إنتاجية الهكتار من القمح

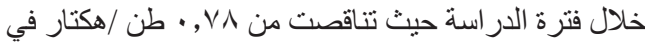

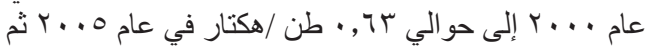

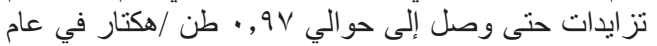

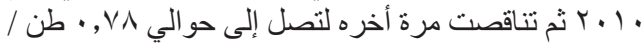

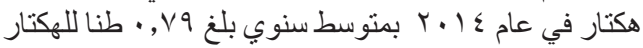
خلال فترة الدر اسة.

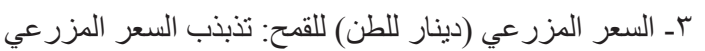

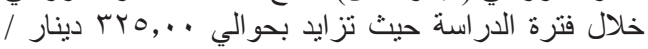

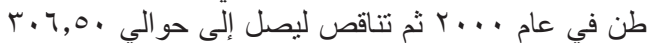

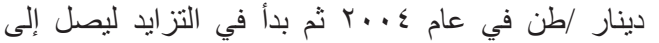

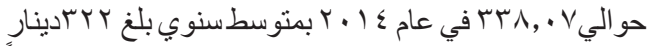

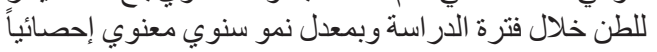

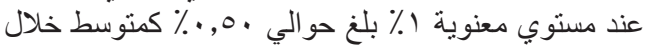

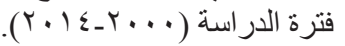
ثانياً: كمية الاستهلاك القومي من القمح وأهم العو امل الاقتصادية

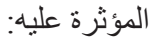
يبين الجدول رقمة ب تز ايد كمية الاستهلاك القومي من القمح

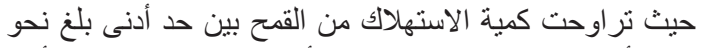

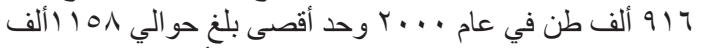

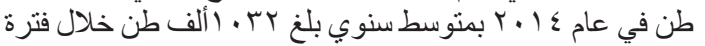

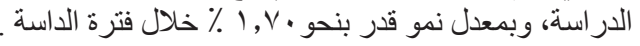
ونظراً لأن التغير في كمية الاستهلاك القومي للقمح هي

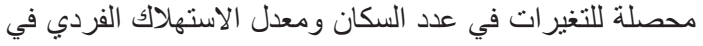
السنة و الدخل القومي يمكن در اسة كل من:
لاختيار المتغيرات التفسيرية ذات التأثير الأفضل والاقوي علي الأني

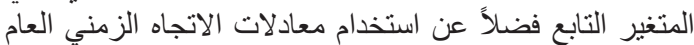

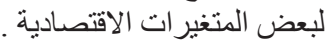

واعتمد البحث على البيانات المنشورة خلال الفترة الفترة

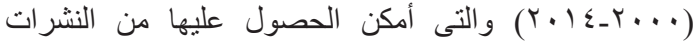

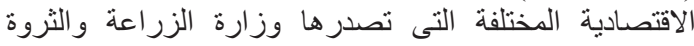

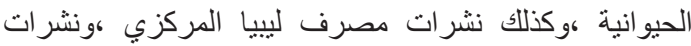

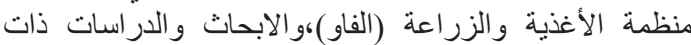
الصلة على الشبكة الدولية للمعلومات (الانترنت الانت).

النتائج و المناقشة

أولاً: كمية الإنتاج المحلي من القمح وأهم العوامل الاقتصادية

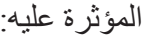

يبين الجدول رقم ا نز ايد كمية الإنتاج المحلي من القمح حيث

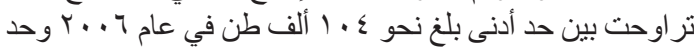

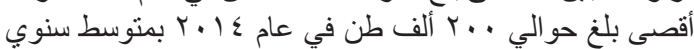

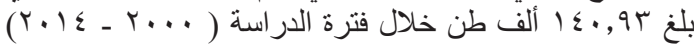

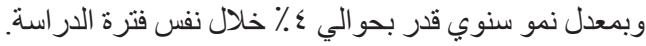
ويمكن در اسة أهم هذه العو امل كما يلي:

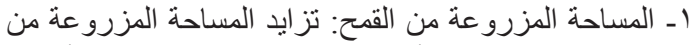

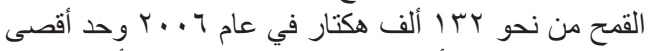

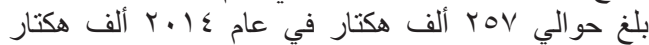

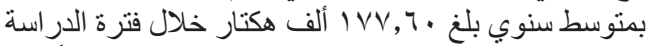

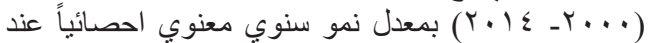

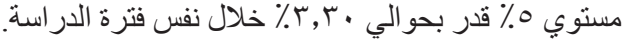

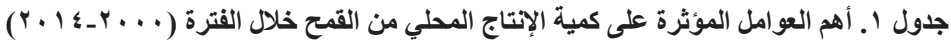

\begin{tabular}{|c|c|c|c|c|}
\hline السعر المز رعي دينار/طن & الهكتارية طن/ الإنتاجية & المساحة المزروعة ألف هكتار & كمية الإنتاج ألف & السنة \\
\hline rro,.. & $\cdot, \vee \wedge$ & $17 \cdot, 91$ & 1ro,... & $r \ldots$ \\
\hline Tוo,.. & Vฯ., & $170, r \leq$ & Iro, . & $r \ldots l$ \\
\hline . & $\cdot, \vee \wedge$ & $17 ., 0 \mathrm{~V}$ & Iro,... & $r \ldots r$ \\
\hline r.,... & $\cdot, V$ & $|7 \varepsilon, \varepsilon|$ & Iro, . & $r \ldots r$ \\
\hline r. & $\cdot, V \vee$ & o. & Iro,... & $r \ldots \varepsilon$ \\
\hline .,.,., & חד, • & $170, \ldots$ & $1 \cdot \varepsilon, \ldots$ & r..o \\
\hline rir,.. & $\cdot, \vee \vee$ & Ir, . & $1 \cdot \varepsilon, \ldots$ & $r \ldots r$ \\
\hline ז,... & $\cdot, \vee q$ & $1 \leq \ldots, \ldots$ & $1 \cdot \varepsilon, \ldots$ & $r \ldots v$ \\
\hline . . . & $\cdot, \wedge$ & $1 \leqslant r, \ldots$ & $1.0, \ldots$ & $r \ldots \Lambda$ \\
\hline . . . & $\cdot, \wedge$. & .... . . & $1 \cdot 7, \ldots$ & $r . . q$ \\
\hline סוTr 10 & $\cdot, 9 \vee$ & $|V|, Y A$ & $177, \ldots$ & r. 1. \\
\hline דॄ, & .,9r & $r 10,79$ & $r \ldots, \ldots$ & $r .11$ \\
\hline ד & $\cdot, \vee \wedge$ & roo, AV & $r \ldots, \ldots$ & $r \cdot 1 r$ \\
\hline r & $\cdot, \vee \wedge$ & roT, Y & $r \ldots, \ldots$ & $r \cdot 1 r$ \\
\hline r & $\cdot, \vee \wedge$ & roT, & $r \ldots, \ldots$ & $r .1 \leq$ \\
\hline . & $\cdot, \vee q$ & IVV, 7 . & $1 \leq \cdot, 9 r$ & المثوسط \\
\hline$* *, 0$ & $\cdot, \wedge$. & *r,r. & $* * \varepsilon, \cdots$ & معدل النمو \\
\hline
\end{tabular}




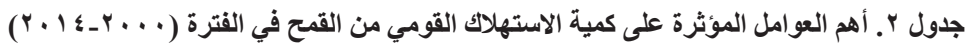

\begin{tabular}{|c|c|c|c|c|c|c|}
\hline كمية الواردات & سعر واردات ليبيا & معدل الاستهلاك & بالأسعار الجاري الفردي & مليون نسمة السكان & كمية الاستهلاك & السنة \\
\hline$\varepsilon r v, r r$ & $19, \ldots$ & $|v|, v$. & Trr & $0, \mu \leqslant$ & $q 1 q, r \leq$ & r... \\
\hline צד & r^,l. & $18 \cdot, q 9$ & rro & $0, \leqslant$ & qrA, Ir & r... \\
\hline$\leq q_{,},+1$ & $\pi, \ldots$ & $|v|, \cdot r$ & $\varepsilon r q$ & $0,0 r$ & $q \leq r, \vee \vee$ & $r \ldots r$ \\
\hline$r \mid \leq, \Lambda \varepsilon$ & $10, \Lambda$. & $I V \varepsilon, V V$ & rrr & 0,71 & $q \wedge \cdot, r$. & $r \ldots r$ \\
\hline r & VY,l. & IVY,.r & 790 & $\bullet, v$. & $9 \wedge 1, . \varepsilon$ & $r \ldots \varepsilon$ \\
\hline $0 \wedge \cdot, r)$ & $\vee \wedge, \varepsilon$. & $\mid V \cdot, T \Lambda$ & vol & $\bullet, \wedge$. & $99 ., 1 \varepsilon$ & r... \\
\hline orr,r. & $\wedge \varepsilon, \vee$. & $I V \cdot, \varepsilon V$ & V^I & 0,91 & $1 \ldots 7,9 \leq$ & $r \ldots r$ \\
\hline Nor,. 9 & $91, \ldots$ & $141,0 r$ & 1.9 & $r, \cdot r$ & $1.1 \leq, \ldots$ & $r \ldots v$ \\
\hline $1.9,97$ & $q \vee, r$. & דו & Nrq & $7,1 r$ & $1 . \leqslant \wedge, \Upsilon$ & $r \ldots \wedge$ \\
\hline$|\wedge 0 \leqslant, \wedge|$ & $1 . r, 4$. & $|V|, \wedge \varepsilon$ & 1०9 & $\neg, Y)$ & $1.74,1$. & r...q \\
\hline $1 v 1 r, 19$ & $1.9,9$. & IVr,Y. & $\wedge ৭ \wedge$ & $7, Y V$ & $1 . \wedge 0,1$. & $r .1$. \\
\hline Arr,. & $118,4$. & $1 V_{0}, \leqslant 4$ & VVY & $\checkmark, Y q$ & $11 \cdot r, r$. & $r .11$ \\
\hline IrVr,Tr & Irr,o. & $\mid \vee \wedge, 01$ & 1.7 & $7, \Upsilon \wedge$ & $11 \times 1, \pi$ & $r .1 r$ \\
\hline$r_{1 .}, \Psi v$ & $\mid r \wedge, \wedge$. & $1 \wedge 1,94$ & nor & $Y, Y V$ & $11 r q, \wedge$. & $r .1 r$ \\
\hline r) & $1+0,1$. & $1 \wedge 0, \cdot \varepsilon$ & $\Lambda \leq \vee$ & $q, Y q$ & $110 \wedge, \ldots$ & $r .1 \leqslant$ \\
\hline
\end{tabular}

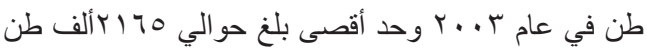

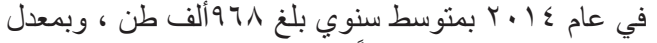

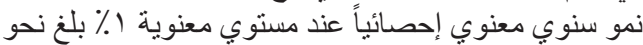

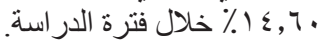

ثالثاً: التحليل الأيكونومثري للعوامل المؤثرة على اقتصاديات محصول القمح في ليبيا:

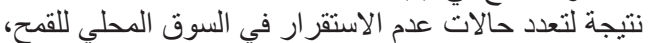

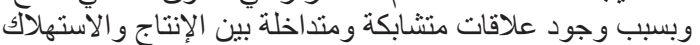

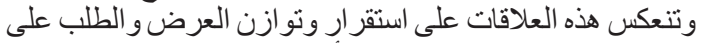

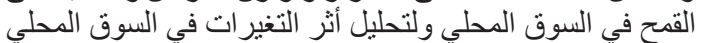

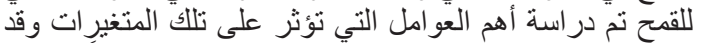

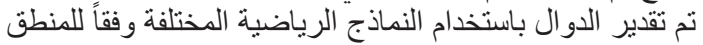

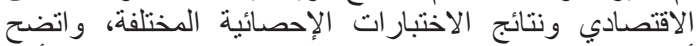

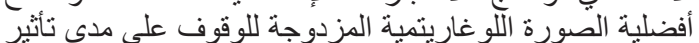
هذه العو امل على الإنتاج المحلي كما يلي: العو امل المؤثرة على الإنتاج المحلي منِ القمح:

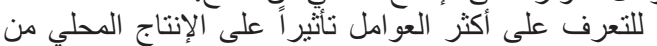

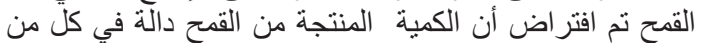

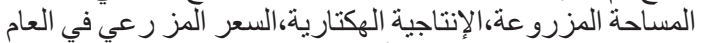

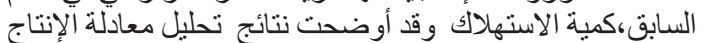

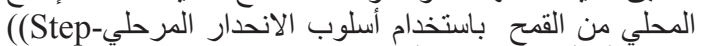

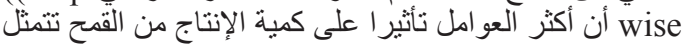

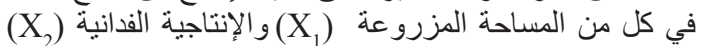
حيث تفسر التغير ات في هذه العو امل نحل نحو

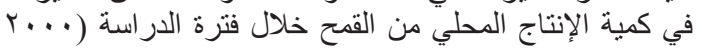

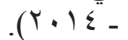
$\mathrm{R}^{2}=\% 95$
$\mathrm{F}=107.52$
D. $\mathrm{W}=1.91$

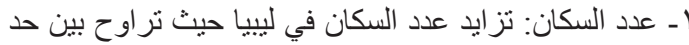

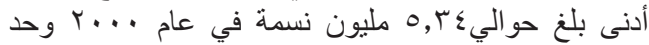

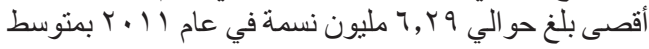

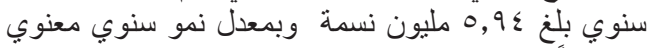

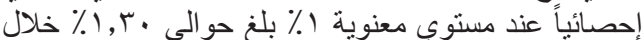

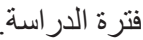

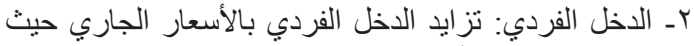

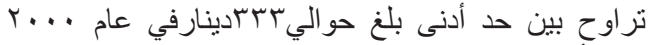

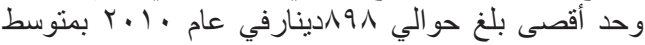

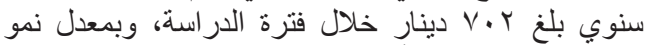

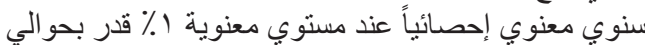

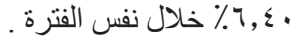

rـ معدل الاستهلاك الفردي كجم/ السنة: تز ايد معدل الاستهلاك

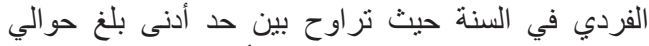

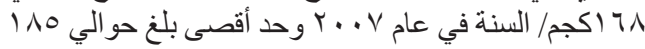

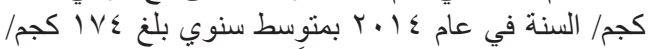

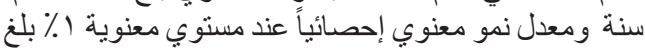
حو الي • ع , • ٪ خلال فترة الدر اسة.

ع ـ سعر و اردات القمح العالمي للطن: تذبذب سعر واردات القمح

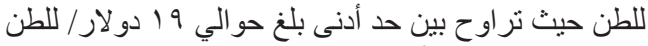

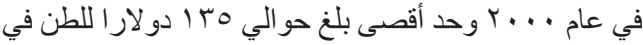

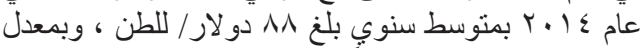

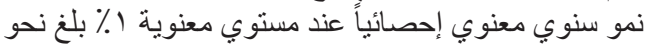

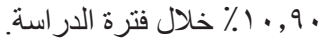

0ـ كمية الواردات من القمح: تذبذبت كمية الواردات من القمح

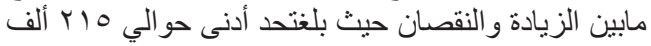




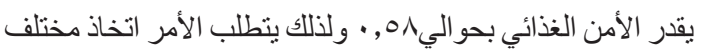

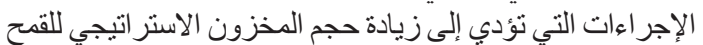

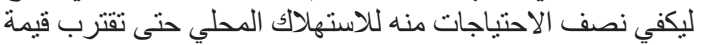
معامل الأمن الغذائي على الأقلّ من الو احد الصحيح.

سادسا: الاتجاهات المستقبلية المتوقعة نحو إمكانية تحقيق الأمن

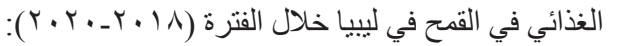

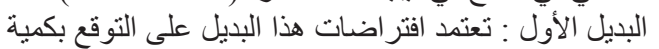

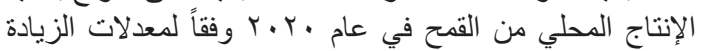

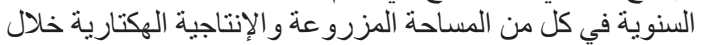

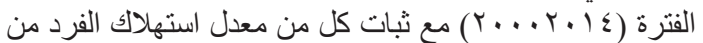

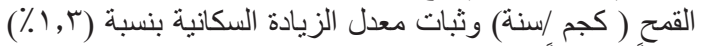

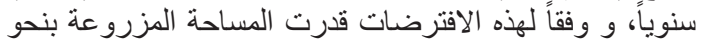

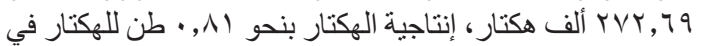

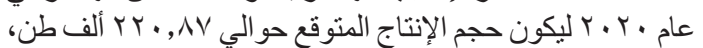

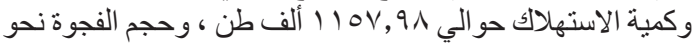

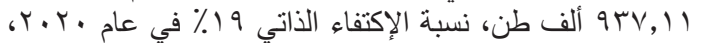

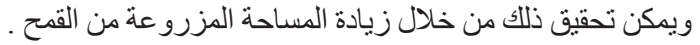

البديل الثاني: تعتمد افتر اضات هذا البديل على التوقع بكمية

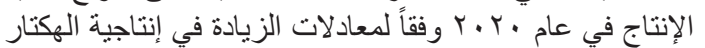

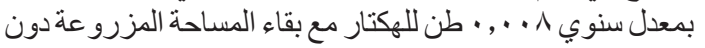

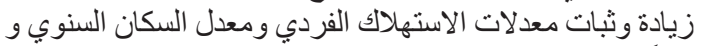

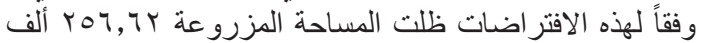

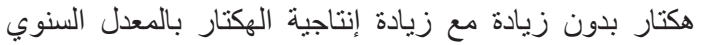

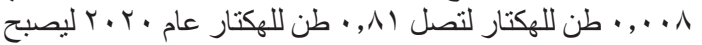

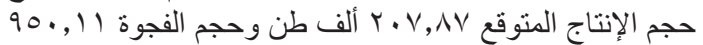

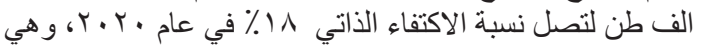

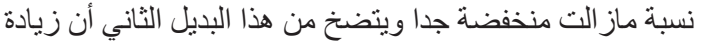

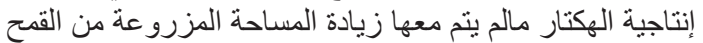

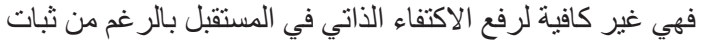
معدل الاستهلاك الفردي ومعدل الزيانيادة في السكان.

البديل الثالث: تعتمد افتر اضات هذا البديل على التوقع بزيادة

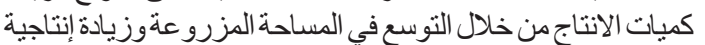

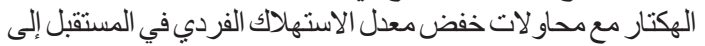

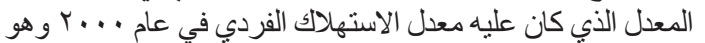

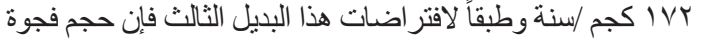

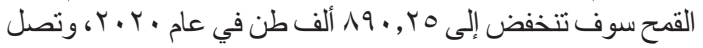

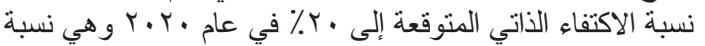
متوسطه في ظل الإمكانيات الاقتصادية الحالية المتاحتة

البديل الرابع: تعتمد افتر اضات هذا البديل على التوقع بزيادة

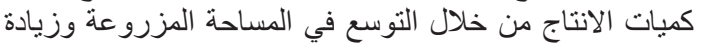

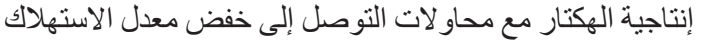

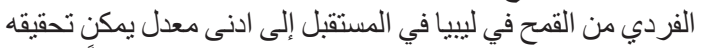

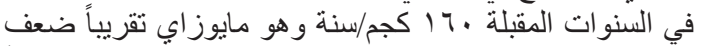

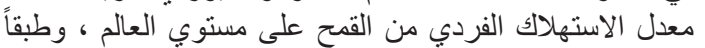

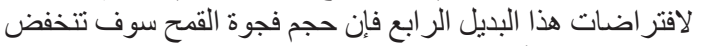

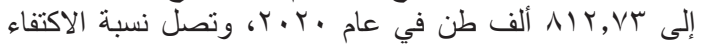

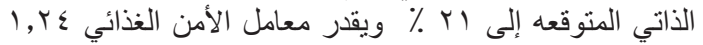

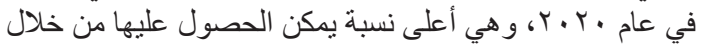

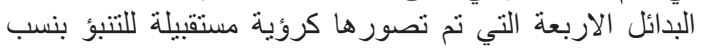

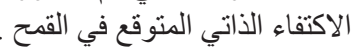

وقد اتفقت إثار ات معالم هذه الدالة مع المنطق الاقتصادي

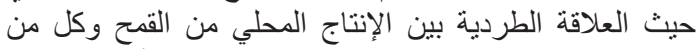

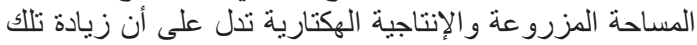

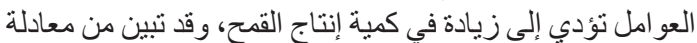

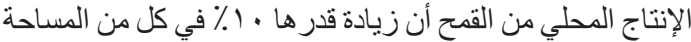

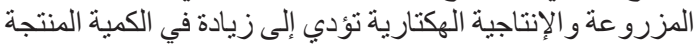

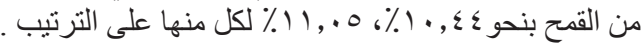

العو امل المؤثرة على الاستهلاك المحلي من القمح:

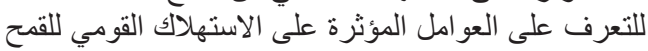

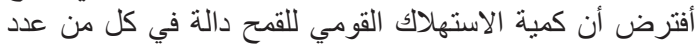

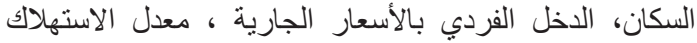

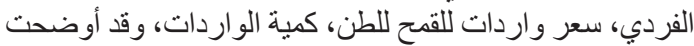

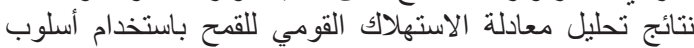

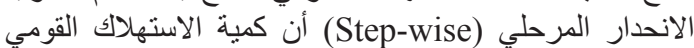

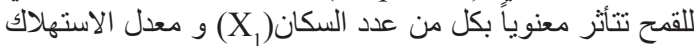

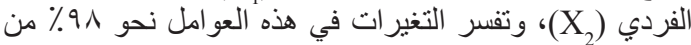

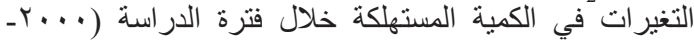

$(13.12)^{*} \quad(5.49)^{*}$

$\mathrm{R}^{2}=\% 98 \quad \mathrm{~F}=291.72 \quad \mathrm{D} . \mathrm{W}=2.34$

وقد اتفقت اشارات معالم هذه الدالة مع المنطق الاقتصادي

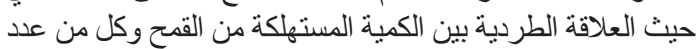
السكان(X) ومعدل الاستهلاكك الفردي (X)

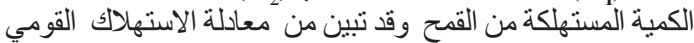

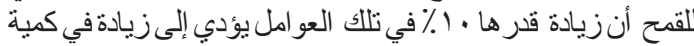

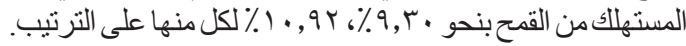

بذلك يتبين أن المساحة المزروعة و إنتاجية الهكتار من أهم الهم

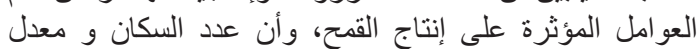

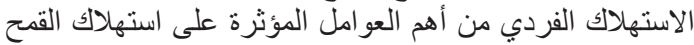
في ليبيا خلال فترة الدر اسة.

رابعاً: الفجوة الغذائية ونسبة الاكتفاء الذاتي في القمح في ليبيا:

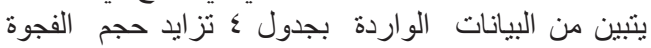

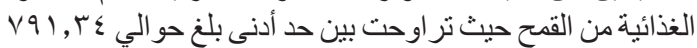

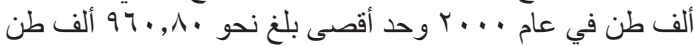

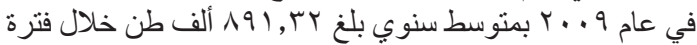

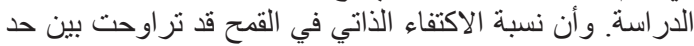

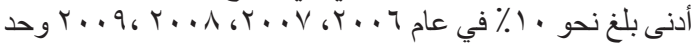

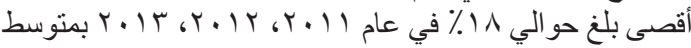
سنوي بلغ ء ا ٪ خلال فترة الدراسة.

خامسا: معامل الأمن الغذائي للقمح في ليبيا خلال فترة الدراسة

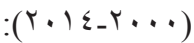

تتر اوح قيمة معامل الأمن الغذائي للقمح بين الصفر و الو احد

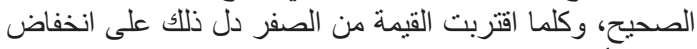

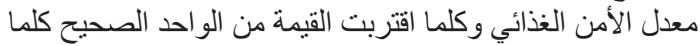

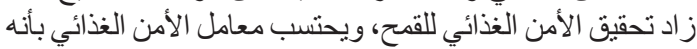

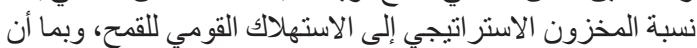

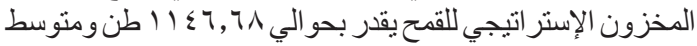

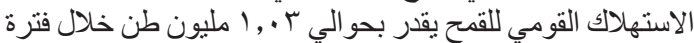

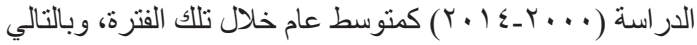




\begin{tabular}{|c|c|c|c|c|c|c|}
\hline QTV, VV & $\Lambda V, O V$ & TR,AT & $V \cdot r$ & I.T,Y, & $0,9 \leqslant$ & متوسط \\
\hline$\cdots 1 \leqslant, 7$. & $* * 1 ., 9$. & ${ }^{* *} \cdot, \varepsilon$. & $* * \eta, \Sigma$. & $* * 1, r$. & $\left.{ }^{* *}\right), V$. & معدل النمو \\
\hline
\end{tabular}

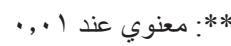

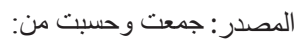

ا - مصرف ليبيا المركزى ، النشرة الإقتصادية ، أعداد متفرقة.

2- United Nations, Food Agriculture Organization, Food Balance Sheets, Different Volumes, Room.

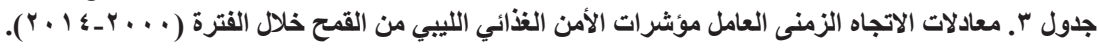

\begin{tabular}{|c|c|c|c|c|c|}
\hline معدل النمو & $\mathbf{F}$ & $\mathbf{R}^{2}$ & $\mathbf{T}$ & المعادلة الم & المتغير \\
\hline${ }^{*}, r$. & $1 \cdot$, rV & $\cdot$, , $\leftleftarrows$ & $r, r)^{*}$ & $\hat{\mathrm{Y}}_{\mathrm{i}}=126.06+6.54 \mathrm{X}_{\mathrm{i}}$ & 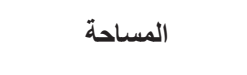 \\
\hline$* * \varepsilon, \ldots$ & $1,1,1 \varepsilon$ &., 0 & $r, \uparrow T^{*}$ & $\hat{\mathrm{Y}}_{\mathrm{i}}=90.13+6.35 \mathrm{X}_{\mathrm{i}}$ & الإلتاج \\
\hline$* * 1, v$ & orre & $\cdot, 99$ & $r \Upsilon, \Upsilon \leq * *$ & $\widehat{\mathrm{Y}}_{\mathrm{i}}=893.68+17.32 \mathrm{X}_{\mathrm{i}}$ & كمية الاستهلاك القومي \\
\hline$*_{* *}^{*}$, , & 15,10 & $\cdot, 0$ & $r, \tau^{* * *}$ & $\widehat{\mathrm{Y}}_{\mathrm{i}}=167.95+0.74 \mathrm{X}_{\mathrm{i}}$ & الاستهلاك الفردي \\
\hline
\end{tabular}

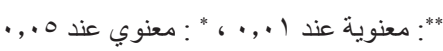

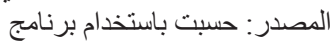

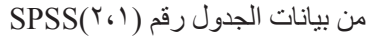

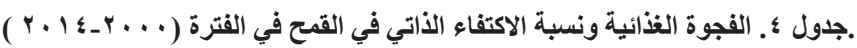

\begin{tabular}{|c|c|c|}
\hline الاكتفاء الذاتي ٪ & الفجوة الغذائية ألف طن & السنة \\
\hline $1 \leqslant$ & $\vee q 1, r \leq$ & r... \\
\hline ir & $\Lambda \cdot r, 1 r$ & $r \ldots r$ \\
\hline ir & $\wedge \curlywedge \wedge, \vee \vee$ & $r \ldots r$ \\
\hline r & $100, r$. & $r \ldots r$ \\
\hline$\pi$ & $107, \cdot \varepsilon$ & $r \ldots \varepsilon$ \\
\hline 11 & $\wedge \wedge 7,1 \leqslant$ & r... \\
\hline 1. & $9 \cdot Y, 9 \leq$ & $r \ldots r$ \\
\hline 1. & $91 \cdot, \ldots$ & $r \ldots r$ \\
\hline 1. & $9 \leqslant r, \uparrow$. & $r \ldots \lambda$ \\
\hline 1. & $97 \cdot, \wedge$. & $r \ldots q$ \\
\hline 10 & 919,1 . & $r \cdot 1$. \\
\hline 11 & q.r.,. & $r .11$ \\
\hline 11 & $941,7$. & $r \cdot I r$ \\
\hline 11 & १५৭,^. & $r \cdot 1 r$ \\
\hline iv & $90 \wedge, \ldots$ & $r \cdot 1 \leq$ \\
\hline$r \cdot r, q Y$ & q, q & إجمالي \\
\hline $1 \leq$ & Aq1,rr & متوسط \\
\hline
\end{tabular}

المصدر: جمعت وحسبت في الجدول رقم(1، Y ).

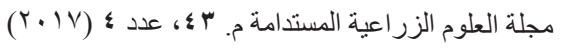




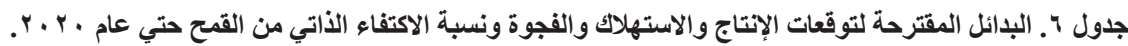

\begin{tabular}{|c|c|c|c|}
\hline$r \cdot r \cdot$ & $r \cdot 1 \leq$ & 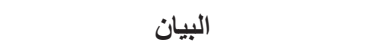 & 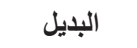 \\
\hline $7, \leqslant 7$ & $\uparrow, r \uparrow$ & عدد السكان مليون نسمة & البديل الأول \\
\hline TVY,Tq & roT,Tr & المساحة المزروعة ألف هكتار & \\
\hline$\cdot, \wedge)$ & $\cdot, \vee \wedge$ & الإنتاجية الهكتارية طن /ه & \\
\hline$r r_{\cdot}, \lambda V$ & $r_{\cdots}, \ldots$ & كمية الإنتاج ألف طن & \\
\hline $110 \mathrm{~V}, 91$ & $1101, \ldots$ & كمية الاستهلاك ألف طن & \\
\hline $9 r v, 11$ & $90 \wedge, \ldots$ & الفجوة الف طن & \\
\hline 19 & iv & \% الاكتفاء الذاتي & \\
\hline 189 & 110 & معدل الاستهلاك الفردي كجم /سنة & \\
\hline roT,Tr & rot,Tr & المساحة المزروعة ألف هكتار & البلديل الثاني \\
\hline$\cdot, \wedge)$ & $\cdot, \vee \wedge$ & الإنتاجية الهكتارية طن /ه & \\
\hline$r \cdot V, \wedge V$ & $r \cdot ., \cdot$ & كمية الإنتاج ألف طن & \\
\hline $110 \mathrm{~V}, 91$ & $1101, \ldots$ & كمية الاستهلاك ألف طن & \\
\hline $90 ., 11$ & $90 \wedge, \ldots$ & الفجوة الف طن & \\
\hline 11 & IV & \% الاكتفاء الذاتي & \\
\hline 189 & 110 & معدل الاستهلاك الفردي كجم /سنة & \\
\hline TVY,79 & roT,Tr & المساحة المزروعة ألف هكتار & البديل الثالث \\
\hline$\cdot, \wedge)$ & $\cdot, \vee \wedge$ & الإتتاجية الهكتارية طن /ه & \\
\hline$r r_{\cdot}, \Lambda V$ & $r \cdot ., \cdot$ & كمية الإنتاج ألف طن & \\
\hline $1111,1 \mathrm{r}$ & $1101, \ldots$ & كمية الاستهلاك ألف طن & \\
\hline$\wedge_{9}, r_{0}$ & $90 \wedge, \ldots$ & الفجوة الف طن & \\
\hline r. & iv & \% اكتفاء الذاتي & \\
\hline IVT & 110 & معدل الاستهلاك القردي كجم /سنة & \\
\hline rVY,Tq & roT,Tr & المساحة المزروعة ألف هكتار & \\
\hline$\cdot, \wedge 1$ & $\cdot, \mathrm{VA}$ & الإنتاجية الهكتارية طن /ه & البديل الرابع \\
\hline$r r_{\cdot}, \Lambda V$ & 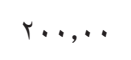 & كمية الإنتاج ألف طن & \\
\hline 1.r., & $1101, .$. & كمية الاستهلاك ألف طن & \\
\hline$\Lambda ו r, V T$ & $90 \wedge, \cdots$ & الفجوة الف طن & \\
\hline r) & iv & \% اكتفاء الذاتي & \\
\hline 17. & 110 & معدل الاستهلاك الفردي كجم /سنة & \\
\hline
\end{tabular}

مجلة العلوم الزراعية المستدامة م. ب ؟ ع عدد ؛ (Y.IV) 


$$
\begin{aligned}
& \text { ه- وزارة الزر اعة والثروة الحيوانية، نشرة أسعار المستهلك للمنتجات } \\
& \text { الزر اعية، أعداد متفرقة. } \\
& \text { 1- مصرف ليبيا المركزى ، النشرة الإقتصادية ، أعداد متفرقة. }
\end{aligned}
$$

7- United Nations ,Food Agriculture Organization, Production Year Book, Different Volume, Room.

8- United Nations, Food Agriculture Organization, Food Balance Sheets, Different Volumes, Room

(Received :27/12/2017;

accepted:5/2/2018)

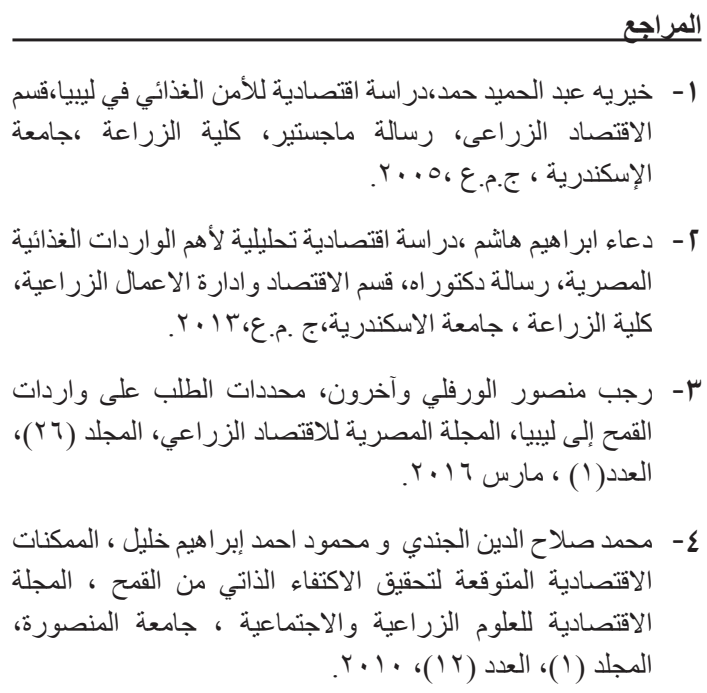

\title{
The Expected Economic Possibilities for Achieving Food Security of Libyan Wheat
}

\author{
Zahra. S.E. Saleh, Nashwa El-Tatatwy, Mounira Taha EL - Hazek and EL-Hussein \\ EL - Seify \\ Department of Economics and Agribusiness, Faculty of Agriculture, Alexandria University \\ Alexandria, Egypt
}

W HEAT is a major strategic commodity in Libya. Wheat is the main item in Libyan meals especially for low income people. Total production of wheat lags behind domestic consumption. The wheat imports amounts to about $\left.r_{-}\right)$million ton. The problem of the study is the disequilibrium between production and domestic consumption. The research aims at shedding the light on the current situation of wheat in Libya by studying the production, consumption, foreign trade, self-adequacy rate, food security factor. The research concerns also with estimating the possibility of achieving food security factor of wheat until the year $r \cdot r \cdot$. To achieve the objectives, To achieve the objectives, the study relied on published secondary data and the Central bank of Libya, food and agriculture organization of the united nation (FAO), and some web sites on the internet. As regards the methodology, the study applied both descriptive and statistical analysis methods particularly simple linear regression equations and multiple regression equations in both the linear and double-log forms, using step-wise regression analysis. The study concerns with determination the factors affecting both wheat quantity produced and domestic consumption in Libya. The study concerns with studying ₹ assumed scenarios for increasing food security factor and choosing the best one.

Keywords: Food Security, Libya, Forecasting, Wheat, Self-sufficiency, Consumption, Production 